\title{
Sensitization to Low Dose 5-Fluorouracil
}

\author{
SUBSEQUENT ENHANCEMENT OF ITS SYSTEMIC
}

ANTITUMOR EFFECT IN THE RAT

\author{
Rudolf E. Falk, Mark Hardy, Leonard Makowka, \\ Julita Teodorczyk-Injeyan, and Judith A. Falk, Department of Surgery \\ and Pathology, University of Toronto, Ontario, Canada M5S 1A8
}

A B S T RACT This report describes a novel method of immunochemotherapy; the active immunization to the drug 5-fluorouracil (5-FU) with enhanced antitumor activity resulting from its subsequent systemic administration. Two metastasizing carcinomas in the Fischer strain (F344) rat have been used: a chemically induced bladder carcinoma (FBCa) and a spontaneous mammary adenocarcinoma (MACa). Both tumors grow rapidly and result in $100 \%$ mortality within $10 \mathrm{wk}$ of implantation. Neither tumor is sensitive to systemic 5-FU alone. Intradermal sensitization to 5-FU before FBCa tumor implantation, followed by 5-FU administered systemically, resulted in significant tumor regression and improvement in survival with eradication of all tumor and cure in $20 \%$ of animals. A similar antitumor effect was observed with the MACa. A comparable drug effect was observed when methotrexate sensitization was given before FBCa implantation followed by systemic MTX. Specificity to the sensitizing drug was demonstrated by the lack of effect of sensitization with either 5-FU or MTX unless followed by systemic therapy with the requisite sensitizing agent. Sensitization to 5-FU has also been assessed after FBCa implantation followed by resection of the local tumor. Resection was performed after distant tumor metastases had occurred, and was followed by systemic 5-FU therapy. Whereas tumor resection alone failed to cure any animal, sensitization to 5-FU increased cure rate fourfold over animals receiving sys-

Dr. Falk is a career investigator of the Medical Research Council of Canada. Dr. Makowka is a research fellow of the Medical Research Council of Canada and a recipient of a Schering Scholarship Award from the American College of Surgeons. Address reprint requests to Dr. R. E. Falk, Department of Surgery, University of Toronto, Ontario, Canada M5S 1 A8.

Received for publication 29 June 1981 and in revised form 2 April 1982. temic 5-FU alone. Antibody to 5-FU in the sera of sensitized animals has been suggested by an immunoenzymatic staining technique and its specificity confirmed in a radioimmunoassay. It is postulated that a combination of the systemic agent and the antibody elicited to it by sensitization produces the significant antitumor effect observed. The antitumor effect observed with this new approach to immunochemotherapy warrants further experimental and clinical study.

\section{INTRODUCTION}

5-Fluorouracil (5-FU) $)^{1}$ was synthesized in $1957(1)$, and since then has become an established antineoplastic agent used clinically in the treatment of various human solid tumors (2-6). The biochemical mechanisms of action for 5-FU have been studied extensively (7-9) with particular emphasis on thymidylate synthetase inhibition (10) and incorporation of 5-FU into RNA (11). Metabolic modulation to enhance either of these two actions has failed to show a causal link with therapeutic efficacy (11-13) and the relative importance of each remains controversial. Neither mechanism excludes an antitumor effect separate from these antimetabolic actions.

The concept that 5-FU acts primarily as a cytotoxic drug affecting rapidly dividing cells has lead to the use of high doses that are active against not only tumor cells, but also cells of the gastrointestinal mucosa and the hematopoietic system $(14,15)$. It has been assumed that 5-FU is immunosuppressive because of the inhibitory effects seen at these high doses (15). Studies of the effect of 5-FU and other fluorodinated pyrimidines

${ }^{1}$ Abbreviations used in this paper: F344, Fischer strain rat; FBCa, methylcholanthrene-induced bladder carcinoma; 5-FU, 5-fluorouracil; MACa, spontaneous mammary adenocarcinoma; MTX, methotrexate; PBS, phosphate-buffered saline. 
on the rodent immune response have been conflicting (16). Merrit and Johnson (17) demonstrated significant augmentation of the murine immune response when 5-FUDr (the deoxyriboside of 5-FU) was given before antigen administration. Conversely, however, 5-FU and 5-FUDr produced immunosuppression when given after antigen administration. Blomgren et al. (18) have demonstrated that delayed hypersensitivity may be augmented by 5-FU. Uy et al. (19) failed to show immunosuppression by either 5-FU or 5-FUDr of the mouse anti-sheep erythrocyte response. Similar lack of immunosuppression has been demonstrated in rabbits (20). The disparate effects of 5-FU on rodent immune responses can be attributed to differences in dosage and in timing of administration in relation to antigen stimulation. These factors are often of critical importance in the immune response to any agent.

It is therefore conceivable that, with particular dosage and timing, 5-FU could augment antitumor responses. We had noted that in some patients with accidental extravasation of 5-FU, an Arthus reaction occurred at that site upon further administration of 5FU at a separate intravenous site. This suggested that an immunoglobulin G (IgG)-mediated immune response to 5-FU had developed in these patients. In certain of the above patients who received lower doses of 5-FU because of toxicity at conventional doses, therapeutic efficacy of chemotherapy appeared increased. In fact, it was observed in two of these cases that at subsequent autopsy they were histologically tumor free, despite diffuse intraabdominal metastases of the tumor at earlier surgery. These incidental clinical observations raised the possibility that the efficacy of 5FU was not dependent only on the cytotoxic effect on tumor cells.

An experimental model was designed to elucidate the basis of these clinical observations. Rats were treated with various low doses of intradermal 5-FU before and after tumor implantation, after which moderate doses of the same drug were administered systemically. This design was meant to test the possibility that immunologic sensitization to the drug could be achieved by low dose intradermal injection, resulting in a subsequent altered systemic effect.

This report describes: $(a)$ the requirements for immunological sensitization to the drug, $(b)$ the markedly increased antitumor effect of 5-FU on two metastasizing rat tumors seen after sensitization, $(c)$ the ability of methotrexate (MTX) to produce a similar effect, $(d)$ the drug specificity of the sensitization produced, and $(e)$ evidence by two techniques that circulating antibody to the drug is present in the sera of the sensitized animals.

\section{METHODS}

Chemicals. 5-FU was purchased from Hoffmann-La Roche Limited (Vaudreuil, Quebec) and methotrexate (MTX) was purchased from Cyanamid of Canada Limited (Montreal, Quebec). Both drugs were diluted with sterile $0.9 \%$ saline before use.

Animals. $250-300-\mathrm{g}$ male and $150-180-\mathrm{g}$ female Fischer strain rats (F344) were purchased from Charles River Laboratories, (Madison, WI) and were caged singly and fed standard rat chow and water ad lib. All animals were allowed to acclimatize for at least $\mathbf{7} \mathbf{d}$ before experimentation.

Tumors. The two tumors used throughout these experiments, a methylcholanthrene-induced bladder carcinoma (FBCa) (kindly supplied by L. Tarranger) and a spontaneous (R3230) mammary adenocarcinoma (MACa) (from the HilfSquibb Medical Research Institute) are both syngeneic tumors in the F344 strain rat. These tumors were maintained in our laboratory by serial passage in F344 rats and a consistent source of tumor was maintained for reference by storage at $-70^{\circ} \mathrm{C}$. The virulence of MACa had been increased by passage through female $F_{1}$ (F344/Wistar-Lewis) hybrids and repassage through female F344 before use. The virulence of FBCa had been similarly increased. Rapid tumor growth is apparent by $14 \mathrm{~d}$ after subcutaneous implantation of a $125 \mathrm{~mm}^{3} \mathrm{FBCa}$ fragment into male rats. At $28 \mathrm{~d}$ postimplantation, the tumor has been fully vascularized and metastatic growth in the lungs is apparent by $42 \mathrm{~d}$, with death resulting by $70 \mathrm{~d}$. A similar fragment of MACa into female rats resulted in rapid tumor growth, apparent by 21 d. At $28 \mathrm{~d}$ postimplantation the tumor was fully vascularized and metastatic growth in the lungs is apparent by $49 \mathrm{~d}$, resulting in death by $70 \mathrm{~d}$.

Surgical resection of FBCa was performed at $21 \mathrm{~d}$ postimplantation of a $125-\mathrm{mm}^{3}$ tumor fragment in the paraspinal position, just posterior to the scapula, with wide excision including the overlying skin. Hemostasis was secured and the incisions closed with 9-mm stainless steel autoclips.

Evaluation of tumor activity. Tumor growth was evaluated weekly using a graduated caliper to measure the three longest dimensions of the tumor at $90^{\circ}$ to each other. An estimate of tumor volume was made using the products of these values. Regression was considered as a decrease in tumor volume lasting for $>14 \mathrm{~d}$. Cure was considered to be survival of $>20 \mathrm{wk}$ with no evidence of local or metastatic tumor. Growth was followed until death, when autopsies were performed to assess metastases.

Drug administration protocols. The timing of drug administration (5-FU or MTX) for the various experiments are outlined in Fig. 1. The low dose intradermal drug referred to as the pretreatment or sensitizing dose, was administered on the shaved dorsal area of the rat. The intravenous drug referred to as the systemic dose was administered via the tail or penile vein.

Antibody detection techniques. Blood samples from animals undergoing pretreatment with intradermal 5-FU were collected weekly from the orbital vein. Heat inactivated sera were tested for the presence of 5-FU binding immunoglobulins using indirect immunoperoxidase staining and $\mathrm{a}$ radioimmunoassay.

Murine thymocytes (Balb/c, 4-6 wk of age) were used as target cells for the immunoenzyme studies. Cells were preincubated for 10-30 min in phosphate-buffered saline (PBS) or RPMI 1640 containing 5-FU at concentrations from 10$500 \mu \mathrm{g} / \mathrm{ml}$. Target cells were then applied to microscope slides by air drying and ethanol/ether mixture fixation. Sera to be tested were applied to the target cells for 10-120 min. The slides were then rinsed twice in PBS for $15 \mathrm{~min}$ and stained with peroxidase-conjugated rabbit anti-rat IgG. Both rat and peroxidase-labeled sera were absorbed before use with murine liver powder and lymphocytes. The sites of specific binding of the peroxidase-conjugated antibody were then demonstrated using Karnovsky's solution (23). 


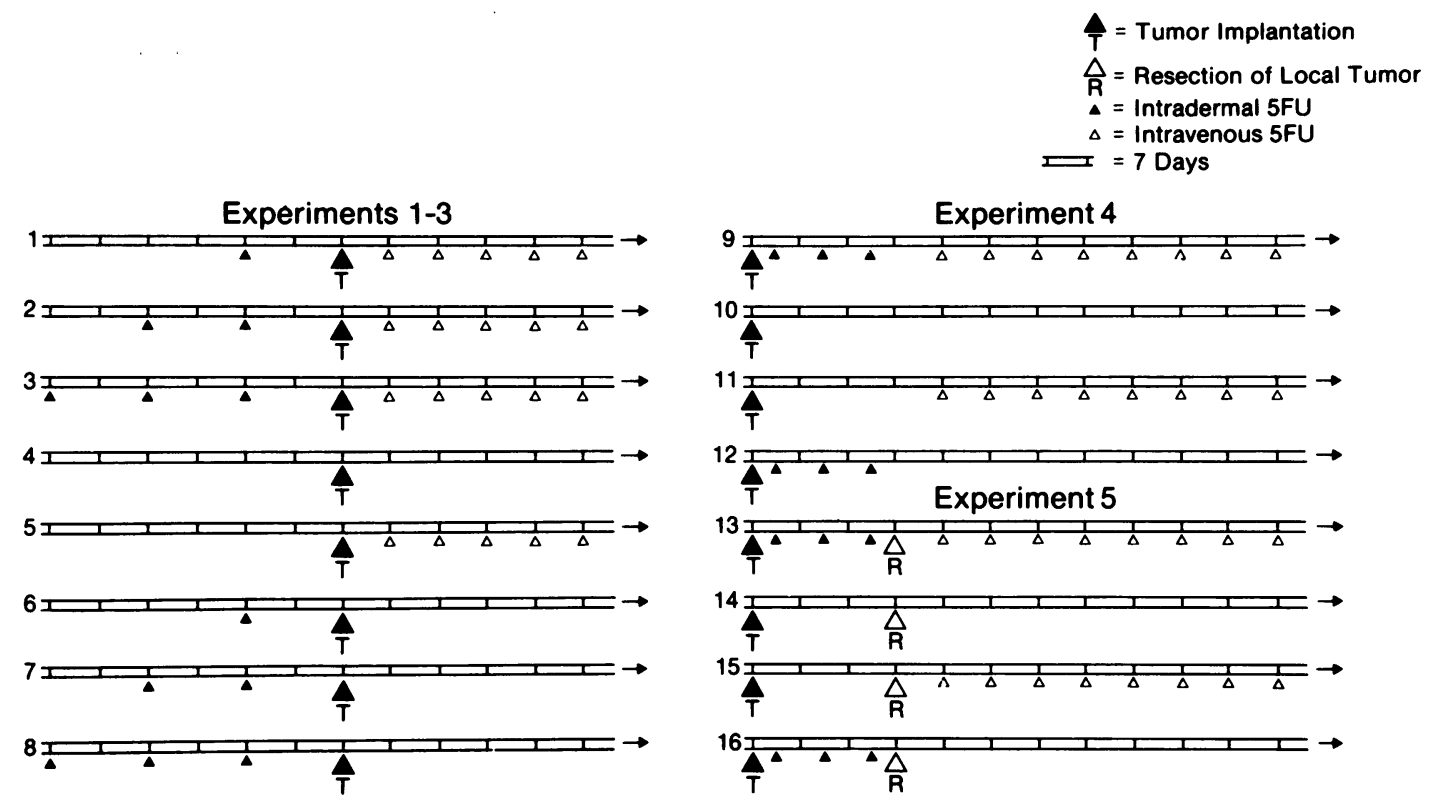

Figure 1 Protocols for chemotherapy administration.

Radioimmunoassay for the detection of anti-5-FU antibodies was performed using nitrocellulose filters as described by Gershman et al. (24) and Schreiber and Raso (25). Briefly 0.25-10 $\mu$ l of sera from control and 5-FU presensitized animals diluted to $400 \mu \mathrm{l}$ with Tris buffer $(0.01 \mathrm{M}, \mathrm{pH} 7.4,0.14$ $\mathrm{M} \mathrm{NaCl})$ were incubated with $10 \mu \mathrm{l}$ of $\left[{ }^{14} \mathrm{C}\right]-5-\mathrm{FU}(400 \mu \mathrm{g} /$ $\mathrm{ml}$ specific activity $482 \mu \mathrm{Ci} / \mathrm{mg}$ ) for $60 \mathrm{~min}$ at $22^{\circ} \mathrm{C}$. Unbound radiolabeled drug was separated by passage through a nitrocellulose filter membrane (GS, $0.22 \mu \mathrm{m}$ ) using the multiple filtration manifold (Millipore Corp., Bedford, MA). Filters were washed with $10 \mathrm{ml}$ of Tris buffer, dried, and the radioactivity of the bound material measured in a scintillation counter.

Statistics. Tumor growth was analyzed using the unpaired Student's $t$ test. Analyses of variance of the animal response was also done. Comparison among the means across time as well as across treatment was assessed using Duncan's multiple range test for variable responses. Mortality and percentage of regression were compared using Chi-square analysis with Yates correction factor. A $P$ value of $<0.05$ was considered significant in all tests.

\section{RESULTS}

Assessment of low doses of 5-FU on tumor growth. The effect of low dose pretreatment with intradermal 5-FU on local tumor growth and the incidence of metastases of FBCa was studied. Male (F344) rats ( $n$ $=60$ ) were divided into three experimental and five control groups. Animals were pretreated with either one dose at $14 \mathrm{~d}(n=5)$ (Fig. 1 , group 1$)$, two doses at 28 and $14 \mathrm{~d}(n=5)$ (Fig. 1, group 2) or with three doses at 42,28 , and $14 \mathrm{~d}(n=5)$ before receiving tumor (Fig. 1, group 3). The pretreatment dose in these experiments consisted of $5-\mathrm{FU} 0.1 \mathrm{mg} / \mathrm{kg}$ body wt in- jected intradermally in the dorsal aspect of the animals. No local cutaneous reactions were observed at the injection site. All three groups then received weekly systemic treatment with $5-\mathrm{FU}(10 \mathrm{mg} / \mathrm{kg}$, i.v. $)$ beginning at $7 \mathrm{~d}$ after tumor implantation.

The control groups for the above experiment received either no treatment $(n=15)$ (Fig. 1, group 4), systemic therapy alone $(n=15)$ (Fig. 1, group 5), or pretreatment with $1(n=5), 2(n=5)$, or $3(n=5)$ doses as for the experimental groups but without systemic therapy (Fig. 1, groups 6-8). There was no significant difference in tumor behavior between the control groups. The tumor growth (Fig. 2) was identical and survival was $20 \%$ by $60 \mathrm{~d}$ and $9 \%$ by $70 \mathrm{~d}$ post FBCa implantation (Table I), and $80 \%$ of animals dying were found to have pulmonary metastases at autopsy. Neither systemic treatment nor pretreatment with intradermal 5-FU was sufficient in itself to produce an antitumor effect. Fig. 2 depicts the growth curves of FBCa for those rats receiving low dose pretreatment and subsequent systemic treatment (Fig. 1, groups 1-3). Pretreatment with one dose of 5-FU, with subsequent systemic treatment demonstrated no difference in the antitumor response compared with the controls. The presensitization treatment effect manifested at two doses before tumor implantation and the decreased rate of tumor growth was highly significant when three doses were used to presensitize animals followed by systemic therapy (Fig. 2A, P $=0.0015$ comparing means across time using Duncan's multiple range test for variable responses). Moreover, $80 \%$ of 

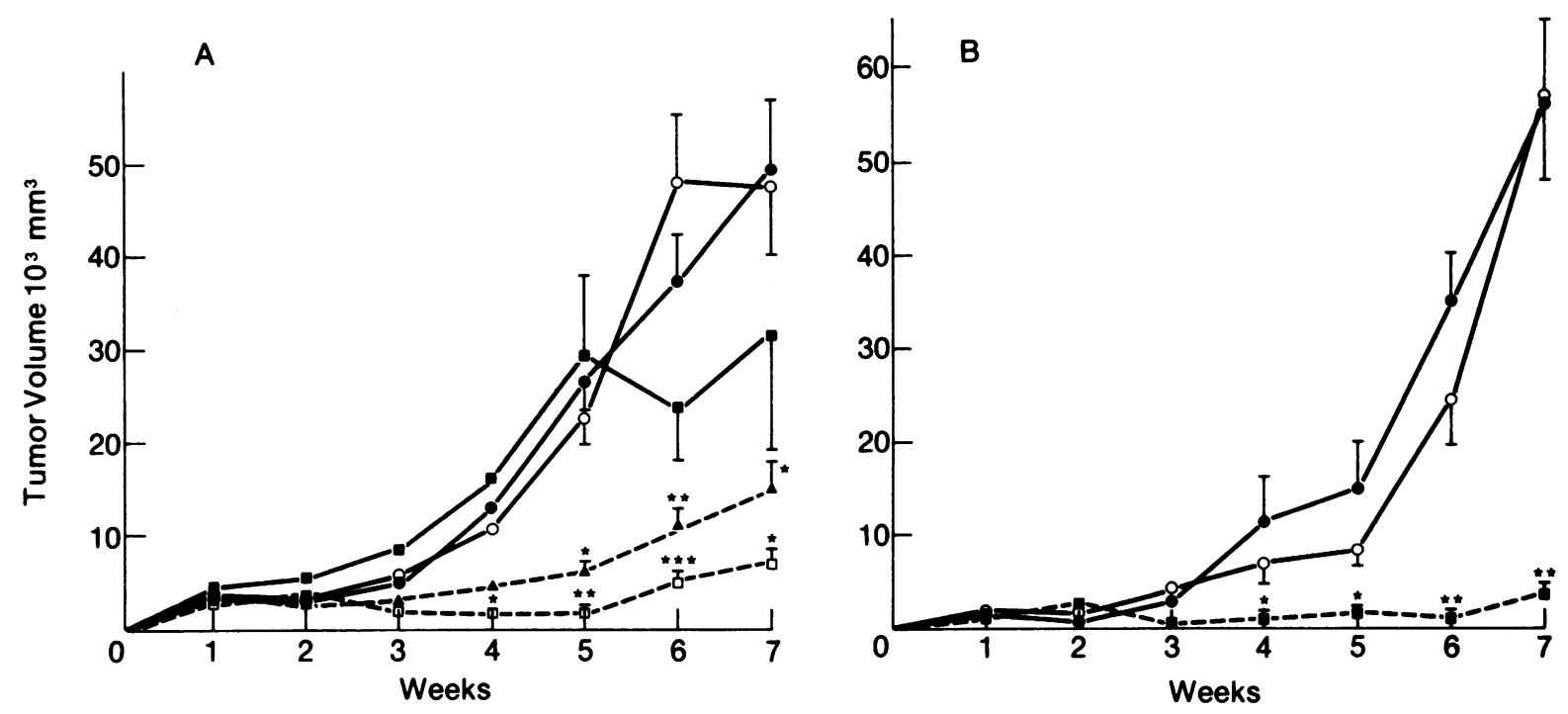

Figure 2 The effect of pretreatment with 5-FU on subsequent growth of FBCa. (A) After implantation of trochar at day 0 , tumor volume was followed in rats having previously received one dose $(\square)$, two doses $(\Delta)$ or three doses $(\square)$ of intradermal $5-F U(0.1 \mathrm{mg} / \mathrm{kg} \mathrm{q} 2 \mathrm{wk})$ and subsequently receiving weekly systemic therapy $(5-\mathrm{FU} 10 \mathrm{mg} / \mathrm{kg}$ i.v.). These volumes (mean \pm SD) were compared with those of rats receiving no treatment $(\bullet)$ and systemic therapy alone $(O)\left(n=5\right.$ for each group) $\left({ }^{\bullet} P<0.05,{ }^{\circ} P<0.01,{ }^{\circ} P P<0.001\right.$ by Student's $t$ test $)$. (B) The marked antitumor effect of sensitization to 5-FU was confirmed in larger groups of animals by using an identical protocol as was followed in $2 \mathrm{~A}$ with three doses of pretreatment (ם) and subsequent systemic therapy compared with no treatment (O) and systemic therapy alone (O) ( $n=20$ for each group). The comparison among the means across time as well as treatment using Duncan's multiple range test for variable responses was as follows: systemic therapy only, 13,461.595; no treatment, 12,225.755; presensitization only, 10,655.400; 3 doses presensitization plus systemic, $4,824.825$.

animals in this group were surviving at $10 \mathrm{wk}$ compared with none in the control (Table I, A).

The results observed for three pretreatment doses plus systemic therapy were then confirmed in a second experiment with increased numbers (Fig. $2 B, n=20$ / group). The tumor growth in control and presensitized animals was compared by analysis of variance and again the three-dose presensitization schedule was highly effective in retarding tumor growth $(P=0.0001$ using Duncan's multiple range test-see legend Fig. 2B). Moreover, $20 \%$ of these animals $(n=5)$ demonstrated marked regression with complete disappearance of the tumor. At $10 \mathrm{wk}$, systemic administration of 5-FU was stopped. $30 \mathrm{wk}$ after tumor implantation these five animals remained free of recurrence and, when sacrificed, showed no metastases.

Evaluation of the antitumor effect of pretreatment on a rat mammary adenocarcinoma. An identical protocol (Fig. 1) but using MACa was used in female F344 rats to determine whether the antitumor effect observed with FBCa was applicable to other tumor models. As seen in Fig. 3, the growth of MACa in the experimental group $(n=10)$ receiving three pretreat- ment doses of $5-\mathrm{FU}(0.1 \mathrm{mg} / \mathrm{kg}$, i.d. $)$ at $42,28,14 \mathrm{~d}$ before MACa implantation, with subsequent weekly administration of systemic therapy $(10 \mathrm{mg} / \mathrm{kg}$, i.v.) beginning $7 \mathrm{~d}$ after tumor implantation was compared with controls receiving either no treatment $(n=10)$, systemic therapy alone $(n=10)$, or three pretreatment doses without systemic therapy $(n=10)$.

No difference in tumor growth was observed among the control groups; no control animals survived $10 \mathrm{wk}$ and $60 \%$ of those dying were demonstrated to have pulmonary metastases. Experimental animals demonstrated a significant inhibition of tumor growth ( $P$ $<0.05$ ) (Fig. 4). Complete regression without recurrence was seen in $20 \%(n=2)$ of experimental animals and 10 -wk survival was $80 \%(n=8),(P<0.01)$ (Table IB). No metastases were found in any of the experimental animals at death.

Evaluating the effect of varying the dosage and timing of pretreatment on antitumor effect. To determine the optimum dose of 5-FU for intradermal pretreatment and schedule of administration necessary for maximum antitumor effect, the following experiment was performed. Experimental groups $(n=5)$ 
TABLE I

Effect on Survival and Cure of Sensitization to 5-FU before Tumor Implantation

\begin{tabular}{rllcl}
\hline Tumor & \multicolumn{1}{c}{ Sensitization } & $\begin{array}{c}\text { Systemic } \\
\text { therapy }\end{array}$ & $\begin{array}{c}10 \text {-wk } \\
\text { survival }\end{array}$ & Cure \\
\hline (A) FBCa & 5-FU 1 dose & 5-FU & $0 / 5$ & $0 / 5$ \\
FBCa & 5-FU 2 doses & $5-F U$ & $1 / 5$ & $0 / 5$ \\
FBCa & 5-FU 3 doses & $5-F U$ & $20 / 25$ & $5 / 25$ \\
FBCa & Nil & Nil & $0 / 25$ & $0 / 25$ \\
FBCa & Nil & 5-FU & $0 / 25$ & $0 / 25$ \\
FBCa & 5-FU 3 doses & Nil & $1 / 25$ & $0 / 25$ \\
& & & & \\
(B) MACa & 5-FU 3 doses & 5-FU & $8 / 10$ & $2 / 10$ \\
MACa & Nil & Nil & $0 / 10$ & $0 / 10$ \\
MACa & Nil & 5-FU & $0 / 10$ & $0 / 10$ \\
MACa & 5-FU 3 doses & Nil & $0 / 10$ & $0 / 10$ \\
\hline
\end{tabular}

(A) The number of $\mathrm{FBCa}$-bearing rats surviving at $10 \mathrm{wk}$ and the number of rats cured of FBCa from those experiments described in Fig. 2.

(B) The number of MACa-bearing rats surviving at $10 \mathrm{wk}$ and the number of rats cured of MACa from the experiments described in Fig. 3.

were given either three pretreatment doses of $(a) 0.05$, (b) $0.1,(c) 0.2 \mathrm{mg} / \mathrm{kg} 5-\mathrm{FU}$, i.d. on $42,28,14 \mathrm{~d}$ before FBCa implantation or six pretreatment doses of $(d)$ 0.05 , and $(e) 0.1 \mathrm{mg} / \mathrm{kg}$, i.d. on $42,35,28,21,14$, and $7 \mathrm{~d}$ before FBCa implantation. Groups of rats that did not receive pretreatment doses of 5 -FU did receive

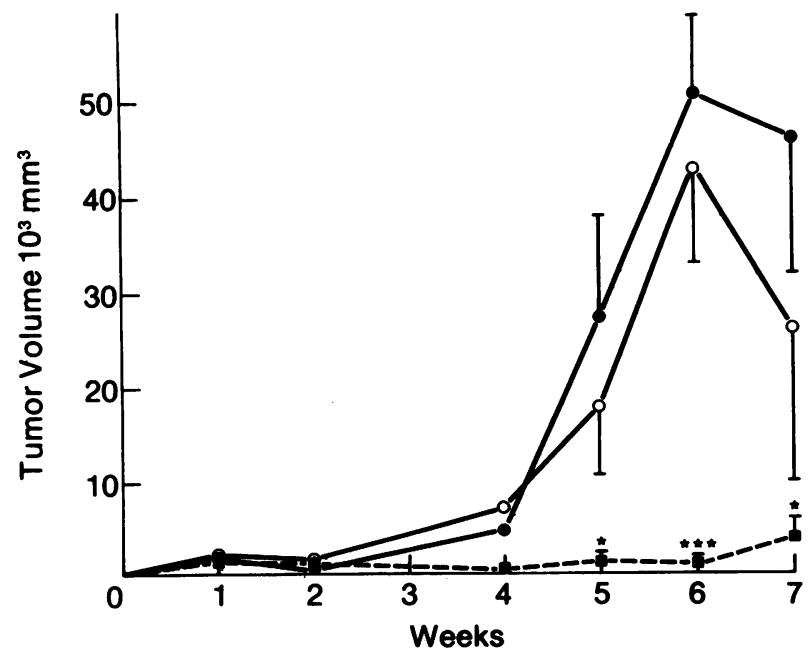

Figure 3 The effect of pretreatment with intradermal 5FU and subsequent systemic treatment with 5-FU on local growth of MACa. Tumor growth was followed in rats receiving three pretreatments $(5-\mathrm{FU} 0.1 \mathrm{mg} / \mathrm{kg}$ i.d. q $2 \mathrm{wk}$ ) before tumor implantation and systemic therapy (5-FU 10 $\mathrm{mg} / \mathrm{kg}$ i.v. q $\mathbf{l}$ wk) afterwards $(\square)$. The volumes (mean $\pm \mathrm{SE}$ ) were compared with those of rats receiving no treatment (๑) and systemic therapy alone (O) $(n=10$ for each group) $\left({ }^{\circ} P<0.05,{ }^{\circ} P<0.001\right)$. drug vehicle (saline) intradermally. The systemic treatment, $10 \mathrm{mg} / \mathrm{kg}$, i.v. weekly from day 7 after FBCa implantation, was kept the same as described in sections 1 and 2.

Control animals received either no treatment $(n$ $=5)$, systemic therapy alone $(n=5)$, or pretreatment as for $(a)-(c)$ above ( $n=5$ /group) but without systemic therapy. There was no significant difference between the controls receiving no treatment, only systemic therapy, or only pretreatment. The tumors grew as previously, (Fig. 2, controls) with no survival past 10 wk and $90 \%$ incidence of pulmonary metastases detectable at autopsy.

Tumor growth was initially inhibited for 2-4 wk in all experimental animals that received both pretreatment and subsequent systemic therapy $(P<0.05)$. However, in all groups except $(a)$ and $(b)$, tumor volume returned to that of controls by $42 \mathrm{~d}$ after implantation and no significant difference was observed in survival or occurrence of metastases. A comparison of the degree of regression observed can be seen in Fig. 4. Three pretreatment doses of $0.05 \mathrm{mg} / \mathrm{kg}$ (group a) produced a greater and more prolonged regression with $60 \% 10$-wk survival $(P<0.01)$. However, no complete regression was observed.

The maximum antitumor effect was observed using $0.1 \mathrm{mg} / \mathrm{kg}$ for three doses on 42,28 , and $14 \mathrm{~d}$ before implantation and was the basis for the protocol selected for both FBCa and MACa in the above experiments (Fig. 1, group 3). Tumor regression was greater and lasted longer in this group $(P<0.05)$. There was $80 \%$ survival at $10 \mathrm{wk}(P<0.01)$ and $40 \%(n=2)$ complete regression.

The effect of intradermal 5-FU after tumor implantation but before systemic administration. To assess the effect of intradermal treatment on existing tumor, experimental animals $(n=10)$ received intra-

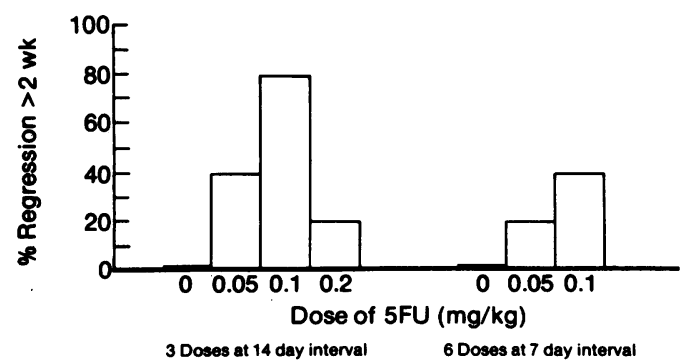

FIgURE 4 The effect of altering the dose and timing of 5FU pretreatment on FBCa regression. The percentage of animals demonstrating tumor regression for $>2$ wk was assessed as those animals in which tumors showed $>20 \%$ reduction in volume for longer than $14 \mathrm{~d}$. This percentage was compared for animals with varying doses of intradermal 5FU and varying intervals between the doses. The zero dose level represents rats that had received intradermal saline as pretreatment. All animals received systemic therapy (5-FU $10 \mathrm{mg} / \mathrm{kg}$ i.v. q $1 \mathrm{wk}$ ) after tumor implantation. 


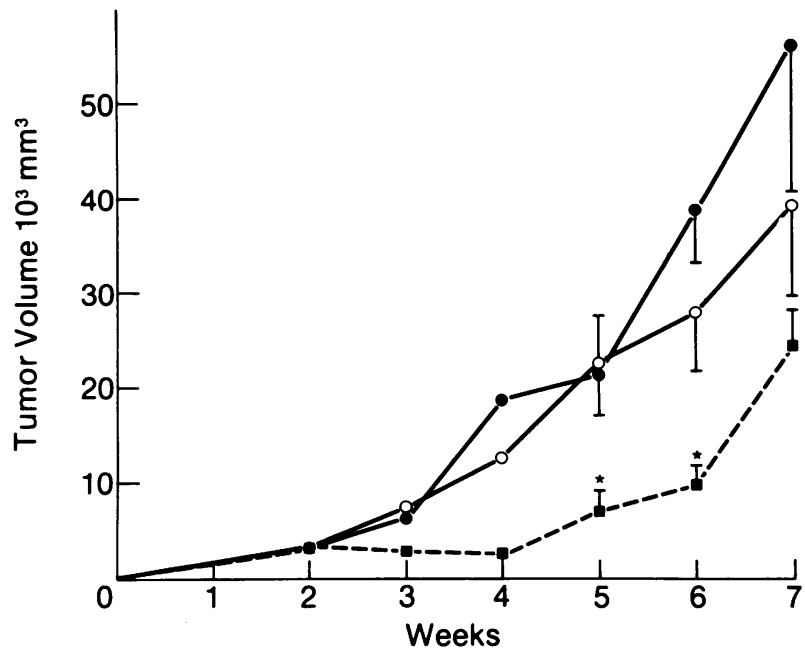

Figure 5 The effect of intradermal 5-FU on tumor growth when administered after implantation of FBCa. Tumor growth was assessed in rats receiving intradermal 5-FU (0.1 $\mathrm{mg} / \mathrm{kg}$ ) on days 3,10 , and 17 after implantation, and then followed by systemic therapy $(10 \mathrm{mg} / \mathrm{kg}$ i.v. q $1 \mathrm{wk}$ ) from day $28(\square)$. The volumes (mean \pm SE) were compared with those of animals receiving no treatment $(\circlearrowleft)$ and systemic therapy $(10 \mathrm{mg} / \mathrm{kg}$ i.v. q $1 \mathrm{wk})$ alone administered from day 28 (O) $\left(n=10\right.$ for each group) $\left({ }^{\circ} P<0.05\right.$ by Student's $t$ test).

dermal 5-FU $(0.1 \mathrm{mg} / \mathrm{kg})$ at 3,10 , and $17 \mathrm{~d}$ after FBCa implantation (Fig. 1 group 9). Subsequent weekly systemic therapy $(10 \mathrm{mg} / \mathrm{kg}$, i.v.) was administered, beginning $28 \mathrm{~d}$ after tumor implantation. Controls received either no treatment $(n=10)$, or systemic therapy alone $(n=10)$ (Fig. 1 groups 10-12). No difference was observed between these controls, and tumor growth and mortality were comparable to previous experiments. In the experimental group no complete regression was observed. However, tumor growth was significantly retarded $(P<0.05)$ (Fig. 5) and the 10wk survival was $70 \%(P<0.01)$ (Table II, A).

The effect of intradermal 5-FU on metastatic spread following resection of the local tumor. The demonstration that intradermal 5-FU pretreatment under the experimental conditions outlined above, reduced tumor growth, and prolonged survival of animals with neoplastic disease, led to an experiment to assess its effect on the development of metastases. The design of this experiment was as described for section 4 with the addition of a surgical resection of the local tumor at $21 \mathrm{~d}$ after FBCa implantation, (Fig. 1 group 13). The control groups for this experiment were resected similarly and were treated as outlined in Exp. 5 (Fig. 1 groups 14-16). Controls receiving no treatment ( $n=10$, group 14) all died within $90 \mathrm{~d}$ of implantation (mean $73 \pm 7 \mathrm{~d}$ ). Local recurrence was not observed in any group; however, in this group lymphatic and pulmonary metastases were extensive. Systemic therapy alone $(n=10$, group 15$)$ resulted in $80 \%$ mortality (mean survival $77 \pm 10 \mathrm{~d}$ ) and the two surviving animals in this group remained free of systemic disease. Intradermal treatment alone $(n=10$, group 16) also resulted in $80 \%$ mortality (mean survival $88 \pm 10 \mathrm{~d}$ ) and the two surviving animals in this group remained free of systemic disease. Intradermal 5-FU plus systemic therapy ( $n=10$, group 13) resulted in significant improvement in survival at 20 -wk postresection $(80 \%, P<0.01)$ (Table IIB) with no evidence of metastatic disease. The only two animals in this group that died (day 80 and day 92 posttumor) had evidence of lymphatic spread but no pulmonary metastases. The other animals are surviving at time of publication.

Assessment of the antitumor effect of intradermal MTX sensitization with subsequent systemic MTX or 5-FU administration. The effect of MTX sensitization on local tumor growth and animal survival in FBCa-bearing rats was studied. Animals were pretreated with three doses of MTX $0.01 \mathrm{mg} / \mathrm{kg}$ body wt, intradermally, at 42,28 , and $14 \mathrm{~d}$ before receiving tumor implantation $(n=5)$. Systemic treatment of weekly MTX $0.75 \mathrm{mg} / \mathrm{kg}$, i.v. began $7 \mathrm{~d}$ after tumor implantation. Control groups received either no treatment $(n=5)$, systemic MTX alone $(n=5)$, or pretreatment intradermal MTX alone $(n=5)$.

The effect of three doses of $5-\mathrm{FU} 0.1 \mathrm{mg} / \mathrm{kg}$, i.d. as sensitization before tumor implantation, followed by weekly 5 -FU systemic therapy $(10 \mathrm{mg} / \mathrm{kg}$, i.v. $)$, was reconfirmed $(P=0.0058)(n=5)$ when compared with controls of rats receiving systemic 5 -FU alone $(n=5)$, and pretreatment 5 -FU alone $(n=5)$. In addition, to assess drug specificity of the sensitization effect, ani-

TABLE II

Effect on Survival and Cure of 5-FU Sensitization following FBCa Implantation and in Combination with Surgical Resection of the Tumor

\begin{tabular}{lllrl}
\hline \multicolumn{1}{c}{ Sensitization } & Resection & $\begin{array}{c}\text { Systemic } \\
\text { therapy }\end{array}$ & $\begin{array}{c}\text { 10-wk } \\
\text { survival }\end{array}$ & Cure \\
\hline (A) 5-FU 3 doses & No & 5-FU & $7 / 10$ & $0 / 10$ \\
Nil & No & Nil & $0 / 10$ & $0 / 10$ \\
Nil & No & $5-F U$ & $0 / 10$ & $0 / 10$ \\
5-FU 3 doses & No & Nil & $0 / 10$ & $0 / 10$ \\
(B) 5-FU 3 doses & Yes & 5-FU & $10 / 10$ & $8 / 10$ \\
Nil & Yes & Nil & $5 / 10$ & $0 / 10$ \\
Nil & Yes & $5-F U$ & $6 / 10$ & $2 / 10$ \\
5-FU 3 doses & Yes & Nil & $5 / 10$ & $0 / 10$ \\
\hline
\end{tabular}

(A) The number of FBCa-bearing rats surviving at $10 \mathrm{wk}$ and the number of rats cured of FBCa from the experiment described in Fig. 5, when rats were sensitized following FBCa implantation.

(B) The number of FBCa-bearing rats surviving at $10 \mathrm{wk}$ and the number of rats cured of FBCa when surgical excision of the tumor (day 21) was combined with 5-FU sensitization following tumor implantation. 
mals sensitized to MTX before tumor implantation were given systemic $5-\mathrm{FU}(10 \mathrm{mg} / \mathrm{kg}$, i.v. weekly) ( $n$ $=5$ ).

There was no significant difference in tumor behavior between any of the control groups, which was the same as described earlier. Neither 5-FU nor MTX systemic therapy alone produced any effect on local tumor growth, survival, or incidence of metastases. 5FU sensitization followed by 5-FU systemic therapy produced marked tumor regression $(P=0.0058$ using Duncan's multiple range test) (Fig. 6) and 60\% survival at $10 \mathrm{wk}(P<0.01)$ (Table III) reconfirming the previous results. MTX pretreatment produced a similar antitumor effect when systemic MTX was subsequently administered. Again, marked tumor regression was observed (Fig. 6) $(P=0.0027$ comparing means across time using Duncan's multiple range test for variable responses) and survival at 10 wk was $80 \%$ ( $P$ $<0.01)$ with one animal showing complete regression and cure of tumor (Table III).

The drug specificity of the pretreatment effect described above was confirmed by the finding that MTX sensitization followed by systemic therapy with 5-FU

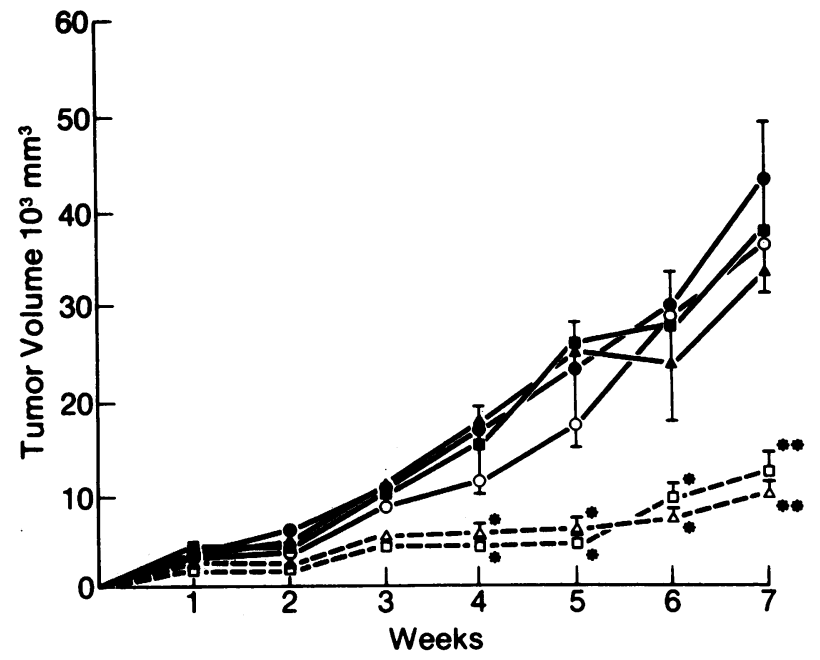

Figure 6 The effect and specificity of pretreatment with either 5-FU or MTX followed by systemic treatment with 5-FU or MTX on FBCa growth. Tumor growth in rats receiving pretreatment with $5-\mathrm{FU}(0.1 \mathrm{mg} / \mathrm{kg}$ i.d. q 2 wk $\times 3)$ before tumor implantation and systemic therapy (5-FU $10 \mathrm{mg} / \mathrm{kg}$ i.v. q $1 \mathrm{wk}$ ) afterwards (口) was compared with those rats receiving pretreatment with MTX $(0.01 \mathrm{mg} / \mathrm{kg}$ i.d. $q 2$ wk $\times 3$ ) before tumor implantation followed by systemic treatment with MTX $(0.75 \mathrm{mg} / \mathrm{kg}$ i.v. q 1 wk) $(\Delta)$. The effect on tumor growth of both pretreatment and systemic treatment with the same chemotherapeutic agent was then compared with the effect of pretreatment with MTX and systemic treatment with 5-FU (O). Tumor growth in all the above groups was also compared with groups of rats receiving no treatment $(\bullet)$, intravenous $5-F U$ only $(\square)$, and intravenous MTX only $(\Delta)$. ( $n=5$ for each group) $\left({ }^{\circ} P<0.05\right.$, $\bullet P<0.01$ by Student's $t$ test).
TABLE III

Survival and Cure of Tumor-bearing Rats Receiving Pretreatment with either 5-FU or MTX

\begin{tabular}{llll}
\hline Sensitization & $\begin{array}{c}\text { Systemic } \\
\text { therapy }\end{array}$ & $\begin{array}{c}\text { 10-wk } \\
\text { survival }\end{array}$ & Cure \\
\hline 5-FU & 5-FU & $3 / 5$ & $1 / 5$ \\
MTX & MTX & $4 / 5$ & $1 / 5$ \\
MTX & $5-F U$ & $0 / 5$ & $0 / 5$ \\
Nil & Nil & $0 / 5$ & $0 / 5$ \\
Nil & 5-FU & $0 / 5$ & $0 / 5$ \\
Nil & MTX & $1 / 5$ & $0 / 5$ \\
5-FU & Nil & $0 / 5$ & $0 / 5$ \\
MTX & Nil & $0 / 5$ & $0 / 5$ \\
\hline
\end{tabular}

The number of FBCa-bearing rats surviving at $10 \mathrm{wk}$ and the number of rats cured of FBCa from the experiment described in Fig. 7.

failed to produce any effect on tumor growth or animal survival (Fig. 6, $P=0.2979$ ) (Table III).

Assessment of antibody to 5-FU in sera of pretreated animals. Experimental and control sera were tested at least five times by the indirect immunoenzyme staining assay. The experimental sera from rats pretreated with three doses of 5-FU before tumor implantation, demonstrated binding to drug-preincubated murine thymocytes. Granules containing peroxidase positive material were demonstrated intracellularly in $20-30 \%$ of target cells. This was not present in any control serum tested. Unimportant surface staining was observed on occasional target cells regardless of the type of serum applied.

In the radioimmunoassay, sera obtained from rats having received three pretreatment doses of $5-\mathrm{FU}$ at $0.1 \mathrm{mg} / \mathrm{kg}$ demonstrated detectable binding to $\left[{ }^{14} \mathrm{C}\right]-$ 5-FU (Fig. 7). The specificity of the binding was tested using sera from animals pretreated with MTX. These sera showed no increase in binding to 5-FU compared with background levels determined when normal rat serum was used in the assay.

\section{DISCUSSION}

It has been demonstrated, in two rapidly growing tumors, FBCa and MACa, that local tumor growth can be significantly retarded by low doses of intradermal 5-FU before tumor implantation when combined with subsequent systemic intravenous therapy at moderate doses. Prolonged survival of animals and complete regression of tumors in some animal was observed. 5FU systemic therapy alone was unable to effect growth or mortality in either of these tumors. Sensitization alone also had no antitumor effect; subsequent systemic therapy was critical. Sensitization performed with tumor in situ followed by systemic therapy produced a significant, but less striking, antitumor effect. 


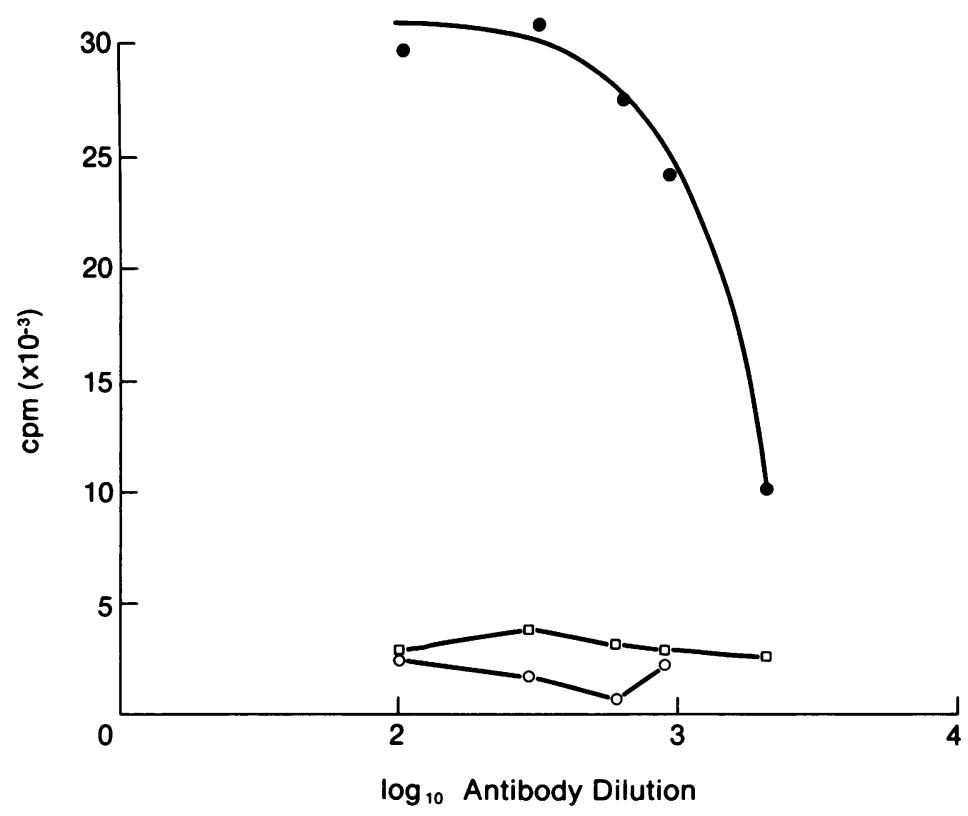

Figure 7 Binding of $\left[{ }^{14} \mathrm{C}\right]-5-\mathrm{FU}$ by sera from sensitized rats. The radioimmunoassay was performed using nitrocellulose filters (Methods). Radiolabeled drug was added to sera from rats who had received pretreatment with 5-FU (0) or MTX (ם). The background binding with normal rat serum $(O)$ is shown.

The kinetics of administration of intradermal 5-FU were found to be critical to the antitumor effect observed. Maximal regression of tumor and prolongation of animal survival were produced by pretreatment with $0.1 \mathrm{mg} / \mathrm{kg} 5$-FU given intradermally for three doses on days 42,28 , and 14 before tumor implantation in comparison with other schedules of pretreatment. Increasing the systemic 5 -FU dose above $10 \mathrm{mg} / \mathrm{kg}$ per wk, i.v., or administering this dose more frequently reduced the antitumor response. The finding that both dosage and timing are integral to the antitumor response, suggests that an immunologic mechanism is involved.

The route of administration was also a factor. Sensitization by intravenous 5-FU given before tumor implantation failed to produce an increased antitumor effect (results not shown). This may be explained by the extremely short plasma half-life of 5-FU given intravenously (26), which may not allow adequate time for immune sensitization to occur.

The effect was not unique to the drug 5-FU. Active sensitization to MTX before systemic MTX therapy could also significantly retard the local growth of FBCa and prolong animal survival. Previous reports demonstrate that MTX can act haptenically, producing an anti-MTX IgG capable of forming drug-protein-antibody complexes in the serum (27). This complex may be the basis of the mechanism by which the observed increased in antitumor effect by presensitization occurs.
It is theoretically possible that the observed antitumor effect with low dose sensitization is purely antimetabolic, and therefore not immunologic or drug specific. This is particularly expected as MTX and 5FU can act synergistically to interfere with the same biochemical step in DNA synthesis (28). If this were so it would be expected that MTX sensitization could also affect the efficacy of subsequent 5-FU systemic therapy. However, no such enhancement occurred. The apparent drug specificity is further supportive of an immune mechanism.

Although clinical hypersensitivity has been described against many chemotherapeutic agents $(5,13$, 29 ), it has not as yet been reported with 5-FU. As well, most observers of this drug sensitivity have viewed the phenomenon from the standpoint of toxicity such as the production of anaphylaxis or circulating immune complex-mediated disease. Our studies suggest that the development of sensitivity by active immunization to a drug may in fact be beneficial. Although hypersensitivity has not been reported with 5 -FU, the physicochemical properties of 5-FU have the prerequisites for haptenic activity (cf. other small molecules such as dinitrophenol). Furthermore, antibody formation has been described to nonfluorodinated pyrimidines, including uracil $(30,31)$.

The presence of an antibody to 5-FU in the sera of 5-FU sensitized animals has been demonstrated by radioimmunoassay and immunoenzymatic localization techniques. The binding to the radiolabeled drug was 
specific to the sensitizing drug. Furthermore, the presence of this anti-5-FU binding activity correlated with the effectiveness of subsequent systemic 5-FU therapy on both local and metastatic tumor growth. The mechanism by which antibody formed against a cytotoxic drug increases tumor cell destruction by that drug remains hypothetical. The therapeutic value of cytotoxic drugs coupled to specific antibody has been studied extensively in the form of passive immunization (32, 33). Administration of drugs chemically linked in vitro to heterogeneous antitumor immunoglobulin may act by increasing target selectivity, with antibody identifying the tumor cell and directing the drug against it. It is also suggested in the literature that the antibody can facilitate the entry of the drug into the tumor cell by altering the tumor cell membrane. The antibody directed against the cytotoxic drug could have a similar mechanism of action on the cell surface as passively administered antibody.

Another possible mechanism of action of antidrug antibody complexes on any cell surface is that this bound complex may cause binding of $\mathrm{Fc}$ receptor bearing cells to the Fc portion of the antibody. Macrophages and neutrophils have such $F c$ receptors, and both are found in substantial numbers in solid tumors. The binding of these phagocytic cells could then lead to tumor destruction. In addition to binding to Fc receptor, the drug-antibody complex(es) could be phagocytosed by and thus activate macrophages with resultant tumor cell destruction.

A novel method of immunochemotherapy with minimal toxicity has been described. Although the exact mechanism of action remains to be defined, the dramatic antitumor responses observed deserve further experimental and clinical investigation.

\section{ACKNOWLEDGMENTS}

We thank Lana Bryant for efficient and cheerful secretarial assistance.

This work was supported by the Medical Research Council of Canada, The National Cancer Institute of Canada, and the Goldie Rotman Memorial Fund.

\section{REFERENCES}

1. Duschinsky, R., E. Pleven, and C. Heidelberger. 1957. The synthesis of 5-fluoropyrimidines. J. Am. Chem. Soc. 79: 4559-4560.

2. Ansfield, F. J., J. M. Schroeder, and A. R. Curreri. 1962. Five years clinical experience with 5 -fluorouracil. JAMA (J. Am. Med. Assoc.). 4: 295-299.

3. Curreri, A. R., F. J. Ansfield, A. McIver, H. A. Waisman, and C. Heidelberger. 1958. Clinic studies with 5-fluorouracil. Cancer Res. 18: 478-484.

4. Heidelberger, C., and F. J. Ansfield. 1963. Experimental and clinical use of fluorinated pyrimidines in cancer chemotherapy. Cancer Res. 23: 1226-1243.

5. Rochlin, D. B., J. Shiner, E. Langdon, and R. Ottoman. 1962. Use of 5-fluorouracil in disseminated solid neoplasms. Ann. Surg. 156: 105-113.
6. Moertel, C. G., and R. J. Reitemeir. 1967. Chemotherapy of gastrointestinal cancer. Surg. Clin. N. Am. 47: 929953.

7. Cohen, S. S., G. Joel, G. Flaks, H. D. Barner, M. R. Loeb, and J. Lichtenstein. 1958. The mode of action of 5-fluorouracil and its derivatives. Proc. Natl. Acad. Sci. U.S.A. 44: 1004-1012.

8. Dannberg, P. B., B. J. Montag, and C. Heidelberger. 1958. Studies on fluorinated pyrimidines. IV Effects on nucleic acid metabolism in vivo. Cancer Res. 18: 329334.

9. Harbers, E., N. K. Chaudhuri, and C. Heidelberger. 1959. Studies on fluorinated pyrimidines. VIII Further biochemical and metabolic investigations. J. Biol. Chem. 234: 1255-1262.

10. Hartmann, K. V., and C. Heidelberger. 1961. Studies on fluorinated pyrimidines. XIII Inhibition of thymidylate synthetase. J. Biol. Chem. 236: 3006-3013.

11. Fernandes, D., and J. Bertino. 1980. 5-Fluorouracilmethotrexate synergy: enhancement of 5-fluorodeoxy uridylate binding to thymidylate synthetase by dihydropteroylpolyglutamates. Proc. Natl. Acad. Sci. U.S.A. 77: 5663-5667.

12. Spiegelman, S., R. Sawyer, R. Nayak, E. Ritzi, R. Stolfi, and $D$. Martin. 1980. Improving the anti-tumor activity of 5-fluorouracil by increasing its incorporation into RNA via metabolic modulation. Proc. Natl. Acad. Sci. U.S.A. 77: 4966-4970.

13. Weiss, R., and B. Salvador. 1981. Hypersensitivity reactions to cancer chemotherapeutic agents. Ann. Int. Med. 94: 66-72.

14. Makinodan, T., G. Santos, and R. Quinn. 1970. Immunosuppressive drugs. Pharmacol. Rev. 22: 189-241.

15. Mitchell, M., and R. DeConti. 1970. Immunosuppression by 5-fluorouracil. Cancer. 26: 884-889.

16. Santos, G. W., and A. H. Owens. 1964. A comparison of the effects of selected cytotoxic agents on allogeneic skin graft survival in rats. Bull. Johns Hopkins Hosp. 116: 327-340.

17. Merritt, K., and A. Johnson. 1963. Studies on adjuvant action of bacterial endotoxins on antibody formation. $J$. Immunol. 91: 266-272.

18. Blomgren, S., W. H. Wolberg, and W. A. Kisken. 1965. Effect of fluoropyrimidines on delayed cutaneous hypersensitivity. Cancer Res. 25: 977-979.

19. Uy, Q., T. Srinivasin, G. Santos, and A. H. Owens. 1966. Effect of selected cytotoxic agents on the primary immune response in mice. Exp. Hematol. 10: 4-6.

20. Sterzl, J. 1961. Effect of some metabolic inhibitors on antibody formation. Nature. (Lond.). 189: 1022-1023.

21. Bross, I. D. J., A. A. Rimm, N. H. Slack, R. K. Ausman, and R. Jones. 1966. Is toxicity really necessary. Cancer. 19: $1780-1784$.

22. Glaser, M. 1979. Augmentation of specific immune response against a syngeneic SV40-induced sarcoma in mice by depletion of suppressor $\mathrm{T}$ cells with cyclophosphamide. Cell. Immunol. 48: 339-345.

23. Graham, R. C., and M. J. Karnovsky. 1966. The early stages of absorption of injected horseradish peroxidase in the proximal tubules of mouse kidney; ultrastructural cytochemistry by a new technique. J. Histochem. Cytochem. 14: 291-302.

24. Gershman, H., E. Powers, L. Levine, and H. Von Vunakis. 1972. Radioimmunoassay of prostaglandins, angiotensin, digoxin, morphine and adenosine $3^{\prime}, 5^{\prime}$-cyclicmono-phosphate with nitrocellulose membranes. Prostaglandins. 1: 407-423. 
25. Schreiber, R., and V. Raso. 1978. Radioimmunoassay for the detection of 5-fluorodeoxyuridine. Cancer Res. 38: 1889-1892.

26. Murkherjee, K. L., and C. Heidelberger. 1960. Studies on fluorinated pyrimidines. IX The degredation of 5fluorouracil-6-C ${ }^{14}$. J. Biol. Chem. 235: 433-437.

27. DaCosta, M., W. Isacoff, S. Rothenberg, and P. Igbal. 1980. Protein-methotrexate-IgG complexes in the serum of patients receiving high dose antifolate therapy. Cancer. 46: 471-474.

28. Cadman, E., R. Heimer, and L. Davis. 1979. Enhanced 5-fluorouracil nucleotide formation after methotrexate administration: explanation of drug synergism. Science (Wash. DC). 205: 1135-1137.

29. Goldberg, N., J. Romolo, E. Austin, J. Drake, and S.
Rosenberg. 1978. Anaphylactoid type reaction in two patients receiving high dose methotrexate. Cancer (Phila.). 41: 52-55.

30. Tanenbaum, S., and S. Beiser. 1963. Pyrimidine-specific antibodies which react with deoxyribonucleic acid. Proc. Natl. Acad. Sci. U.S.A. 49: 662-668.

31. Erlanger, B., and S. Beiser. 1964. Antibodies specific for ribonucleosides and ribonucleotides and their reaction with DNA. Proc. Natl. Acad. Sci. U.S.A. 52: 68-74.

32. Lee, F., and K. Hwang. 1979. Antibodies as specific carriers for chemotherapeutic agents. Cancer Chemother. Pharmacol. 3: 17-24.

33. DeVita, V. T., and H. Busch. 1979. Binding of anticancer drugs to carrier molecules. Methods Cancer Res. 16: $346-380$. 